\title{
Analysis of Transverse Resistive Instability in the Recycler
}

\author{
A. Burov
}

December 21, 2005

\begin{abstract}
Transverse resistive wall instability is analysed for antiprotons in the Recycler Ring. Damper requirements are formulated.
\end{abstract}

\section{Growth Rate and Landau Damping}

\subsection{Coasting Beam}

Coherent instabilities are driven by the real part of impedance. For a coasting beam with insignificant frequency spread, the impedance-driven coherent tune shift at the harmonic number $n$ is given by (see e. g. [1])

$$
\Delta \nu_{Z} \equiv \Delta \omega_{Z} / \omega_{0}=-i \frac{N r_{0} \bar{\beta}}{4 \pi \gamma T_{0}} Z\left(\omega_{b}+n \omega_{0}\right)
$$

where $N$ is the number of particles (pbars), $r_{0}$ - classical radius, $\gamma=E_{0} / m c^{2}$ - relativistic factor, $T_{0}$ - the revolution period, $\omega_{0}=2 \pi / T_{0}, \bar{\beta}$ - the beta-function, and $Z(\Omega)$ - the transverse impedance.

The beam can be naturally stabilized by the Landau damping, which requires sufficient density of particles resonant with the coherent beam motion. Density of the resonant particles is suppressed by the space charge incoherent tune shift, separating coherent and individual betatron frequencies:

$$
\Delta \nu_{s c} \equiv \Delta \omega_{s c} / \omega_{0}=\frac{N r_{0}}{4 \pi \gamma^{2} \varepsilon_{\perp} B}
$$

with $\varepsilon_{\perp}$ as the r. m. s. normalized emittance and $B=\tau_{b} / T_{0}$ is the bunching factor.

Assuming the beam energy spread as a leading source of the single-particle tune spread, via the chromaticity $\xi$ and the slippage factor $\eta=\gamma_{t}^{-2}-\gamma^{-2}$, the r. m. s. spread of the effective incoherent tunes is given by

$$
\Delta \nu_{b}(n) \equiv \Delta \omega_{b}(n) / \omega_{0}=|\eta n-\xi| \delta p / p \quad,
$$

the Landau damping rate is calculated as

$$
\Lambda_{L}=\sqrt{\frac{\pi}{2}} \Delta \omega_{b}(n) x_{n}^{2} \exp \left(-x_{n}^{2} / 2\right)=\sqrt{\frac{\pi}{2}} \Delta \omega_{s c} x_{n} \exp \left(-x_{n}^{2} / 2\right), \quad x_{n} \equiv \Delta \omega_{s c} / \Delta \omega_{b}(n) .
$$

To be specific, the longitudinal distribution was assumed to be Gaussian here; if it is not, the correction is obvious. Note that the dimensionless energy spread $x_{n}$ does not change, if the beam is adiabatically bunched: it depends on the longitudinal phase space density. In other words, growth of the space charge tune shift with the beam bunching is compensated by an equal growth of the momentum spread, so that the dimensionless spread $x_{n}$ does not change. As a consequence, the Landau damping grows linearly with the bunching factor.

When the space charge tune shift is high compared with the coherent tune shift, $\Delta \omega_{s c} \gg\left|\Delta \omega_{Z}\right|$, the stability threshold, i. e. the solution for $\Lambda_{L}=\operatorname{Im} \Delta \omega_{Z}$ is almost independent on the impedance. The stability condition can be approximately presented as 


$$
x \geq x_{\mathrm{th}}, \text { or } \quad|\eta n-\xi| \delta p / p \geq \Delta \nu_{s c} / x_{\mathrm{th}},
$$

where $x_{\mathrm{th}}$ is a numerical factor, $x_{\mathrm{th}} \simeq 3-5$ depending (logarithmically) on the space charge over the impedance tune shifts ratio, and reflecting the particle distribution over the momentum. By the same reason, the stability condition is not sensitive to the bunching factor, when the impedance is space-charge dominated, $\Delta \omega_{s c} \gg\left|\Delta \omega_{Z}\right|$. The stability condition can also be presented in terms of a threshold frequency $f_{t h} \equiv n_{t h} / T_{0}$ :

$$
f \geq f_{t h} \equiv \frac{\left|\xi_{t h}\right|-|\xi|}{|\eta| T_{0}}
$$

where the threshold chromaticity $\xi_{t h}$ is written from (5) as

$$
\xi_{t h}=\frac{N r_{0}}{4 \pi x_{\mathrm{th}} \gamma \varepsilon_{\perp}} \frac{m c^{2} T_{0}}{\varepsilon_{\|}},
$$

with $\varepsilon_{\|}=c \delta p T_{0}$ as the longitudinal r. m. s. emittance, When the chromaticity module is higher than the threshold, $f_{t h}<0$, the beam is stable for any frequency (mode number). If the chromaticity cannot be elevated as high, the beam is going to be unstable at harmonics below the threshold frequency.

Hadron machines with their long bunches are usually dominated by the resistive wall impedance. When the skin depth is smaller than the wall thickness,

$$
Z(\omega)=g Z_{0} R_{0} \delta(\omega) / b^{3}
$$

where $Z_{0}=4 \pi / c=377 \mathrm{Ohm}, R_{0}$ is the machine average radius, $\delta(\omega)=c / \sqrt{2 \pi \sigma \omega}$ is the skin depth, $b$ is the aperture radius, and $g$ is a geometric factor. For round chambers, $g=1$, for flat geometry $g=\pi^{2} / 12 \approx 0.82$ in the vertical direction, and $g=\pi^{2} / 24 \approx 0.41$ in the horizontal. For Recycler, the vertical resistive wall impedance at revolution frequency is calculated as $21 \mathrm{M} \Omega / \mathrm{m}$.

When the synchrotron frequencies are small compared with the coherent tune shift (1), they can be neglected in the stability analysis. For the Recycler, the synchrotron periods are at the range of 1 second, while the instability growth time is typically at least about an order of magnitude shorter. In this case, the tail of the bunch can act back on the head through the multi-turn wake $W(s)$.

\subsection{Why Bunching Can Be Neglected}

Landau damping is a steep exponential function. Beam bunching changes its pre-exponent factor as well as the impedance-related growth rate. However, for space-charge dominated impedances, the exponent is high, and the stability is mostly determined by that, with relatively minor modification by the pre-exponent parameters. Note, that the exponent does not change with the beam bunching, being determined by the phase space density. That is why the beam bunching can be neglected for stability analysis for space charge dominated impedances.

\subsection{Two-Particle Model}

Although the detailed dependence of the instability growth rate $\Delta \omega_{Z}$ on the bunching factor is not so significant, it could be presented here for reader's curiosity, in case of a simple two-particle model. When the bunch is presented as 2 macro-particles:

$$
\begin{gathered}
\Delta \omega_{Z}^{\text {two-part }}=\frac{N r_{0} \bar{\beta}}{4 \gamma T_{0}}(G(0)+\sqrt{G(s) G(-s)}) \\
G(s)=\sum_{k=0}^{\infty} W\left(k C_{0}-s\right) \exp \left(2 \pi i k \nu_{b}\right),
\end{gathered}
$$

where $\nu_{b}=\omega_{b} / \omega_{0}$ is the betatron tune, and $C_{0}$ is the circumference. The plot at Fig. (1) shows slow increase of the growth rate with the bunch squeezing for Recycler's tune $\nu_{b}=0.42$.

These results are supported by more careful numerical analysis, where the beam was represented by much higher number of macro-particles [2], and more recent analytical results [3]. 
Two-Particle Model

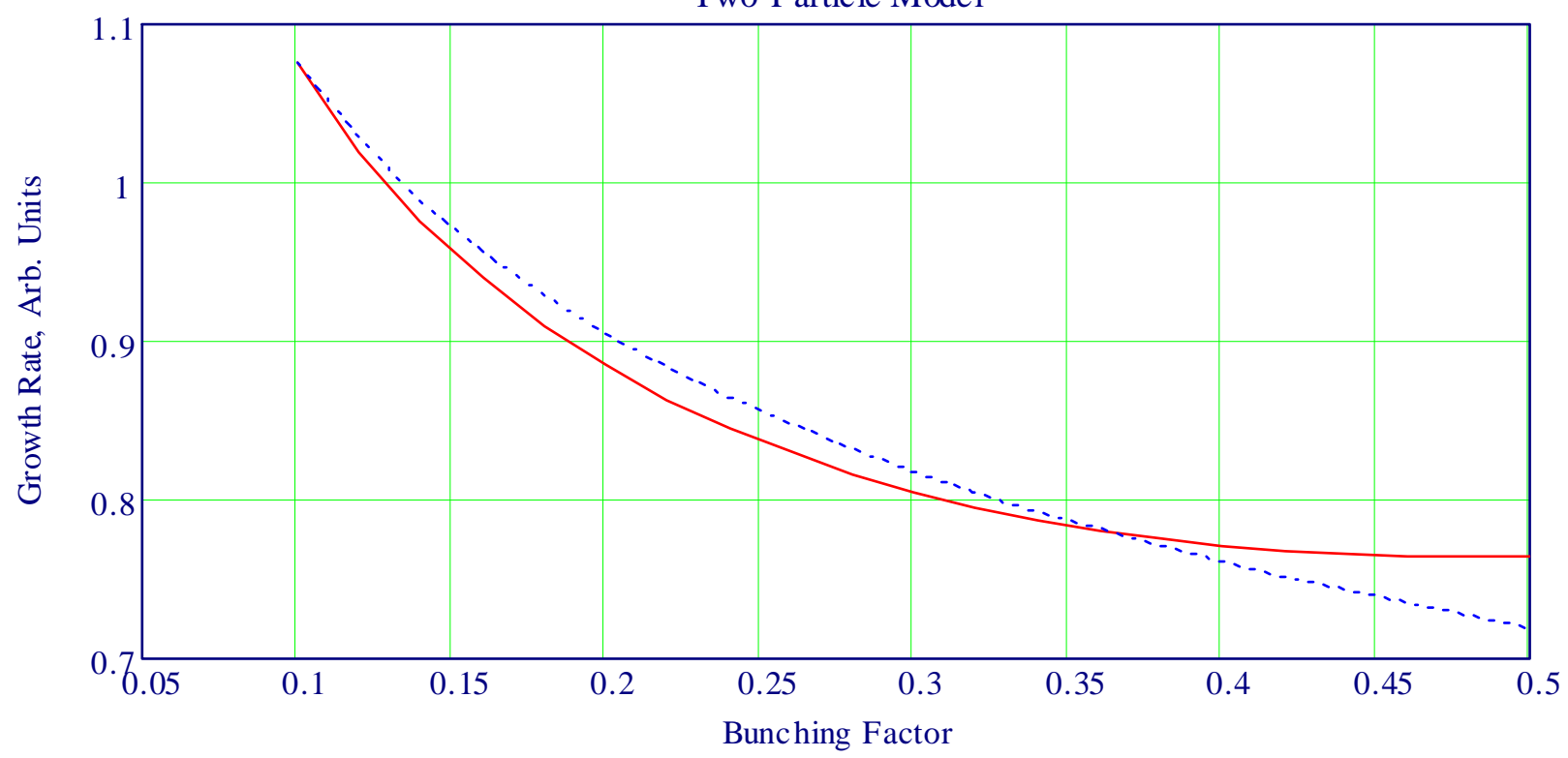

Figure 1: Growth rate of the transverse resistive wall instability for two-particle model: exact result (red) and a fit $s^{-1 / 4}$ (blue).

\subsection{Example: Stability Threshold for Recycler}

When the beam parameters are given, the growth rate and Landau damping can be calculated. Their intersection at the lowest frequency corresponds to the instability threshold. For a given chromaticity, this threshold can be conveniently expressed in terms of effective phase space density

$$
D \equiv \frac{\left(N / 10^{10}\right)}{4 \varepsilon_{\|}[\mathrm{eV} \cdot \mathrm{s}] \cdot 6 \varepsilon_{\perp}[\mathrm{mm} \cdot \mathrm{mrad}]} .
$$

Fig. (2) shows instability growth rate and Landau damping rate for a coasting beam with $N=1.8 \cdot 10^{12}$, $4 \varepsilon_{\|}=50 \mathrm{eV} \cdot \mathrm{s}, 6 \varepsilon_{\perp}=7 \mathrm{~mm} \cdot \mathrm{mrad}, \xi=-6$. As it is seen from the plot, the parameters taken correspond to the instability onset for the most unstable lowest order mode. The effective phase space density here is $D=0.5$, corresponding to $x_{\mathrm{th}}=2.7$. In practice, the instability onset was observed within an interval $0.5 \leq D \leq 0.8$ [4]. Increase of the threshold density over its Gaussian limit $D=0.5$ indicates that the distribution tails are higher than the Gaussian ones.

\section{Digital Damper}

A damper is assumed here to consist of a pickup, pre-amplifier, delay line, analog-digital converter (ADC), notch filter, analog low-pass filter (LPF) and kicker. The pre-amplifier band is assumed here to be broad enough to consider it infinite, so it is simply characterized by the introduced low-frequency damping rate $\Lambda_{0}$. All other elements are considered below.

\subsection{Analog-Digital Converter}

The output signal of ADC goes with a sample frequency $f_{s} \equiv \omega_{s} /(2 \pi) \equiv \tau_{s}^{-1}$, at the time of writing this statement $f_{s}=53 \mathrm{MHz}$, being exactly 588 harmonic of the revolution frequency (to filter out all the 


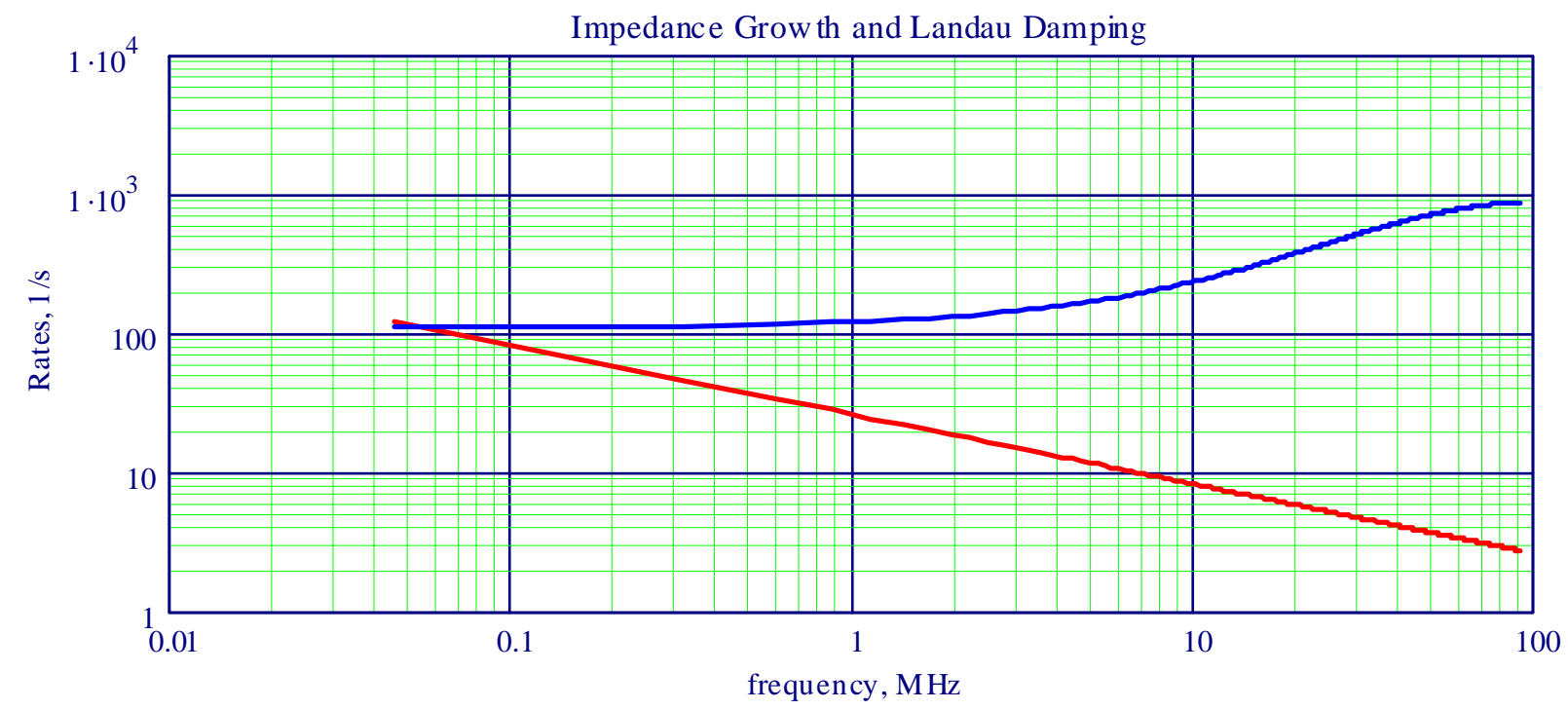

Figure 2: Impedance-driven growth rate (red) and Landau damping (blue), both in inverse seconds, near the threshold. The effective density $D=0.5, x_{\mathrm{th}}=2.7$.

revolution harmonics). Presently, the input signal is detected at $N_{a}=4$ times higher frequency, and then an average of these $N_{a}$ numbers goes as the output. An example of the AD conversion is shown in Fig (3).

The ADC transforms any input frequency into a sequence of all the composite frequencies, shifted from the input one by multiples of the sample frequency. This equidistant sequence of frequencies includes a single one inside an interval $0<\omega<\omega_{s}$, which can be taken as a parameter of the entire set of the cross-talking frequencies. This continuous parameter $\omega$ is referred below as a marking frequency. Incoming frequencies $\omega_{p} \equiv \omega+p \omega_{s}, p=0, \pm 1, \pm 2, \ldots$ are transformed by the ADC into outgoing frequencies $\omega_{q} \equiv \omega+q \omega_{s}, q=0, \pm 1, \pm 2, \ldots$. Let $\widehat{T}$ be the linear operator of the ADC; then, it is straightforward to show that

$$
\begin{gathered}
\widehat{T} \exp \left(-i \omega_{p} t\right)=\sum_{q=-\infty}^{\infty} T_{p, q} \exp \left(-i \omega_{q} t\right) \\
T_{p, q}=\frac{2}{N_{a}} \exp \left[i \omega_{p} \tau_{s}\left(1-\frac{1}{2 N_{a}}\right)\right] \frac{\sin ^{2}\left(\omega_{p} \tau_{s} / 2\right)}{\tau_{s} \omega_{q} \sin \left(\frac{\omega_{p} \tau_{s}}{2 N_{a}}\right)} .
\end{gathered}
$$

Below, it is assumed that the phase factor in the ADC is compensated by a preceding delay line, providing all the matrix elements real:

$$
T_{p, q}=\frac{2}{N_{a}} \frac{\sin ^{2}\left(\omega_{p} \tau_{s} / 2\right)}{\tau_{s} \omega_{q} \sin \left(\frac{\omega_{p} \tau_{s}}{2 N_{a}}\right)} .
$$

With the ADC, the frequency $\omega$ (representing actually the wave length of the beam perturbation) is no longer a good parameter for the beam modes, each consisting of all the composite harmonics. High enough harmonics are strongly damped by Landau damping; thus, they can be neglected and the infinite set of the composite amplitudes being cut.

Let $A_{p}$ be an amplitude of the harmonic $\omega_{p}=\omega+p \omega_{s}$. Were the digital damper the only way for the beam to interact with itself, the time evolution of this harmonic would be described as

$$
\frac{d A_{p}}{d t}=-\Lambda_{0} \sum_{q=-\infty}^{\infty} T_{p, q} A_{q}
$$




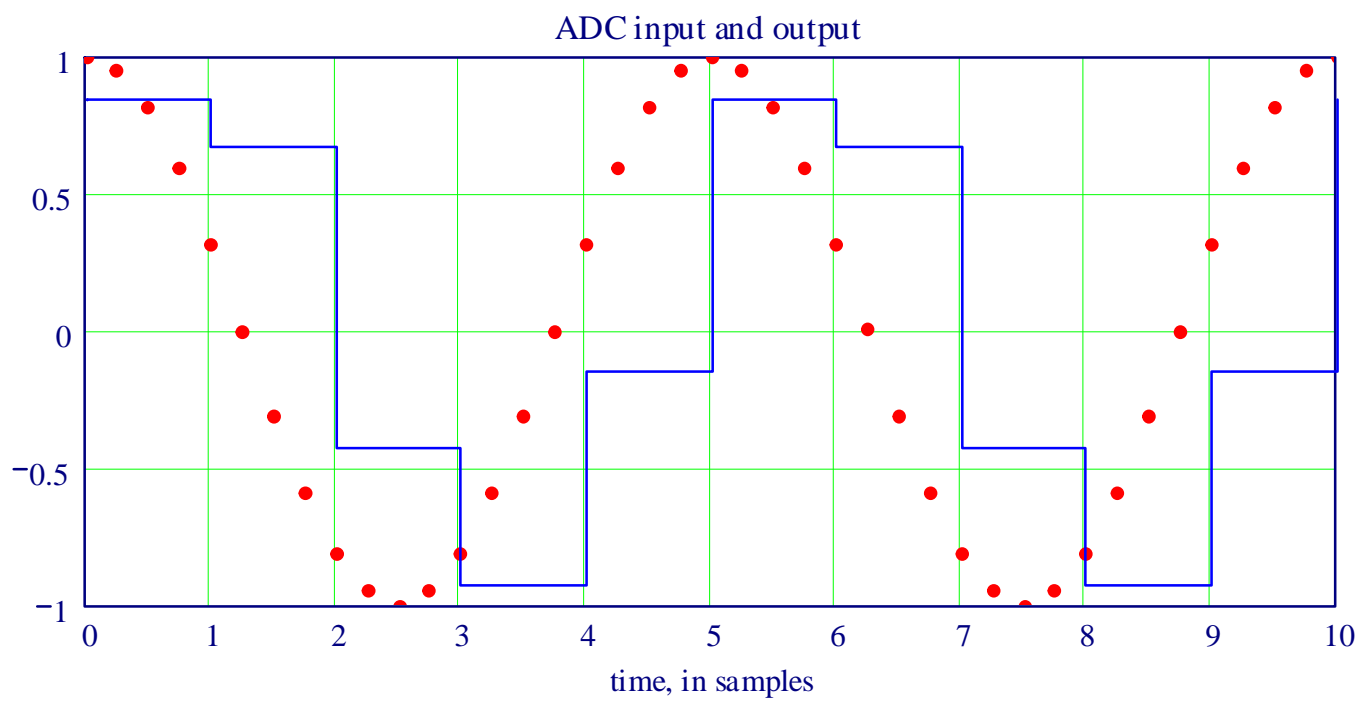

Figure 3: ADC input (red dots) and output (blue steps) for $13 \mathrm{MHz}$ input signal.

with $\Lambda_{0}$ as a low-frequency rate, determined by the pre-amplifier. A solution of this set of linear equations is expressed in terms of eigenvectors, whose eigenvalues are the damping rates of the beam modes. Impedance and Landau damping just add their terms to the matrix diagonal elements:

$$
\frac{d A_{p}}{d t}=-\Lambda_{0} \sum_{q=-\infty}^{\infty} T_{p, q} A_{q}-\left(\Lambda_{L}\right)_{p} A_{p}-i\left(\Delta \omega_{Z}\right)_{p} A_{p}
$$

Note that the matrix $\widehat{T}$ (11) is strongly degenerated: for any finite dimension it is reduced, all its eigenvalues but one are exact zeroes. With impedance, half of these zeroes are getting unstable; they could be stabilized by the Landau damping.

\subsection{Low-Pass Filter}

A filter $\widehat{F}$ transforms the signal as

$$
\widehat{F} \exp (-i \omega t)=F(\omega) \exp (-i \omega t) .
$$

With a filter following the ADC, the ADC matrix elements are modified as $T_{p, q} \rightarrow T_{p, q} F\left(\omega_{q}\right)$. A simplest, first-order, low-pass filter (LPF) is given by an R-C circuit:

$$
F(\omega)=\frac{1}{1-i \omega / \omega_{F}} .
$$

Presently, a high-order LPF provides the filter function as it is shown in Fig. (4), provided by J. Crisp [5]

\subsection{ADC+LPF+Impedance+Landau Damping}

To solve the stability problem with all the four factors taken into account, the impedance and Landau damping rates should be added to the diagonal elements of the ADC+LPF matrix. The instability threshold is calculated as twice higher effective phase space density, $D=1.1$, practically the same in a wide range of the damper's low-frequency damping time $\Lambda_{0}^{-1}$, between 15 and 1500 revolutions. In reality, the beam was 

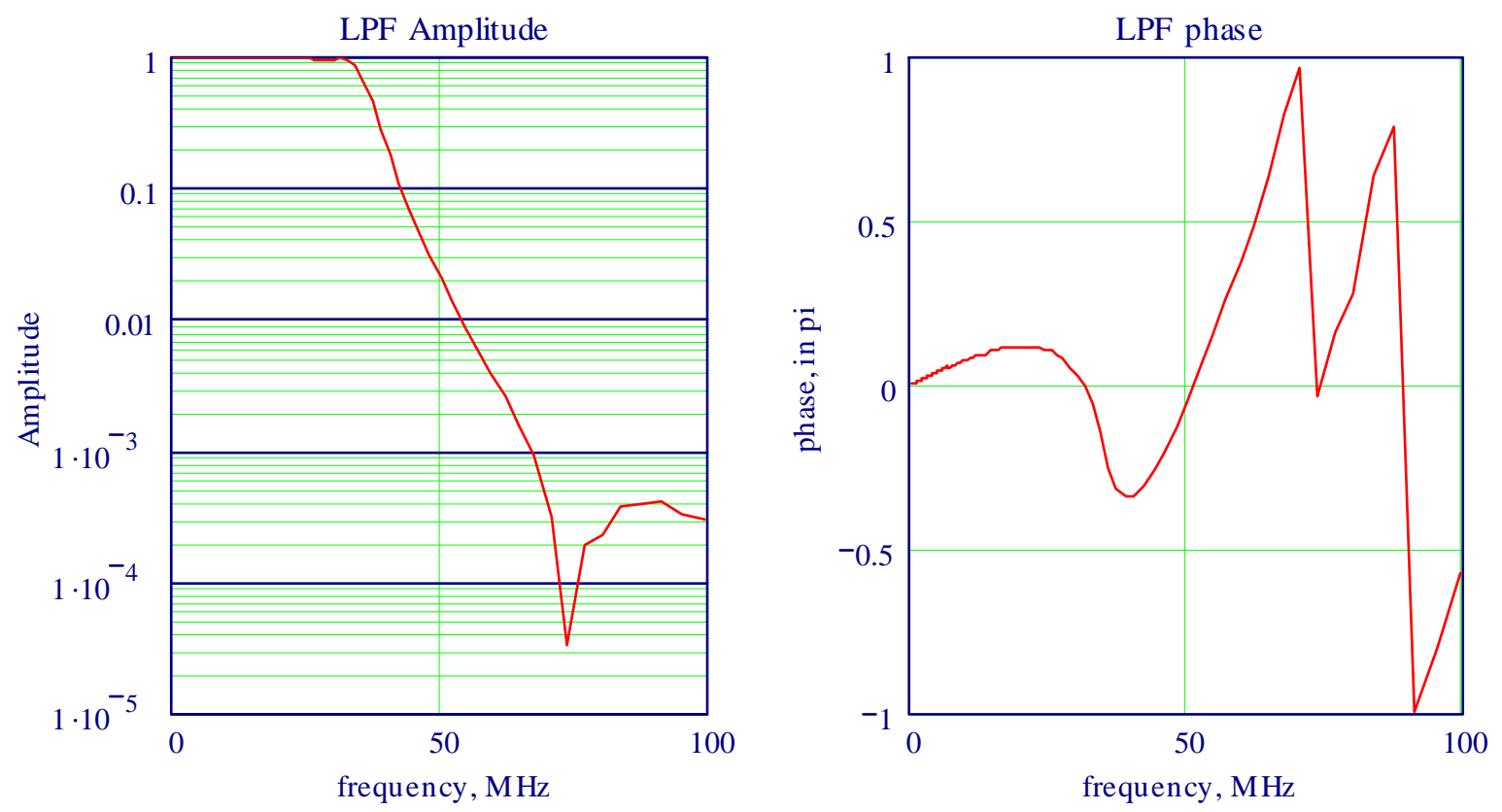

Figure 4: Amplitude and phase characteristics of the LPF of the present damper.

observed as stable up to $D=1.5$ [4], a factor of 2 more than without the damper. Lower thresholds in this model, compared with the observations (by 40\%) most likely result from the Gaussian assumption. The real distribution of the cooled beam is not Gaussian, the real tails are higher and the real thresholds should be higher as well. Some results for a specific threshold case with $N=2.8 \cdot 10^{12}, 4 \varepsilon_{\|}=35 \mathrm{eV} \cdot \mathrm{s}$, $6 \varepsilon_{\perp}=7 \mathrm{~mm} \cdot \mathrm{mrad}$, are presented in Figs. (5), (6).

Fig. (5) shows that for this choice of parameters, Landau damping prevails at $f \gtrsim 35 \mathrm{MHz}$, not far from the Nyquist frequency $f_{s} / 2=26.5 \mathrm{MHz}$.

Fig. 6, left, shows the damping rate for the less stable mode as a function of the marking frequency;clearly, it is the instability threshold. On the right, the average frequency of this less stable mode is calculated, where all the composite frequencies are weighted with amplitudes of their perturbations squared. The curve minimum is close to the Nyquist frequency $26.5 \mathrm{MHz}$.

One more curious observation is that LPF appears to be useless here. The threshold densities with and without it are calculated as identical within a few percent. So, the current LPF does neither good nor bad. The reason is that if the filter is wider than the Landau damping boundary, it makes nothing. Otherwise, it makes the damper inefficient in a frequency range between the filter cut-off and the Landau damping start.

\subsection{Offset of the Sampling Frequency}

Up to this point, the sampling frequency was supposed to be an exact multiple of the revolution frequency, which is needed to suppress all the revolution harmonics by the Notch filter. As a result, all the composite harmonics generated by the ADC are located at the betatron sidebands, and effectively interact with the beam. That is why the cross-talk between the harmonics was taken into account in the above analysis. However, some offset of the sampling frequency from the exact multiple of the revolution one is possible; its limit is determined by the beam centering and other parameters external for this analysis. If this detune exceeds several times the impedance-driven growth rate, then the composite harmonics do not interact with the beam, the damper matrix is diagonal, and the beam should be more stable. Indeed, the cross-talk of the harmonics requires Landau damping to prevail at about Nyquist frequency. Without the cross-talk, Landau damping is needed to prevail at the sampling frequency, which damper just does not see. According to the 


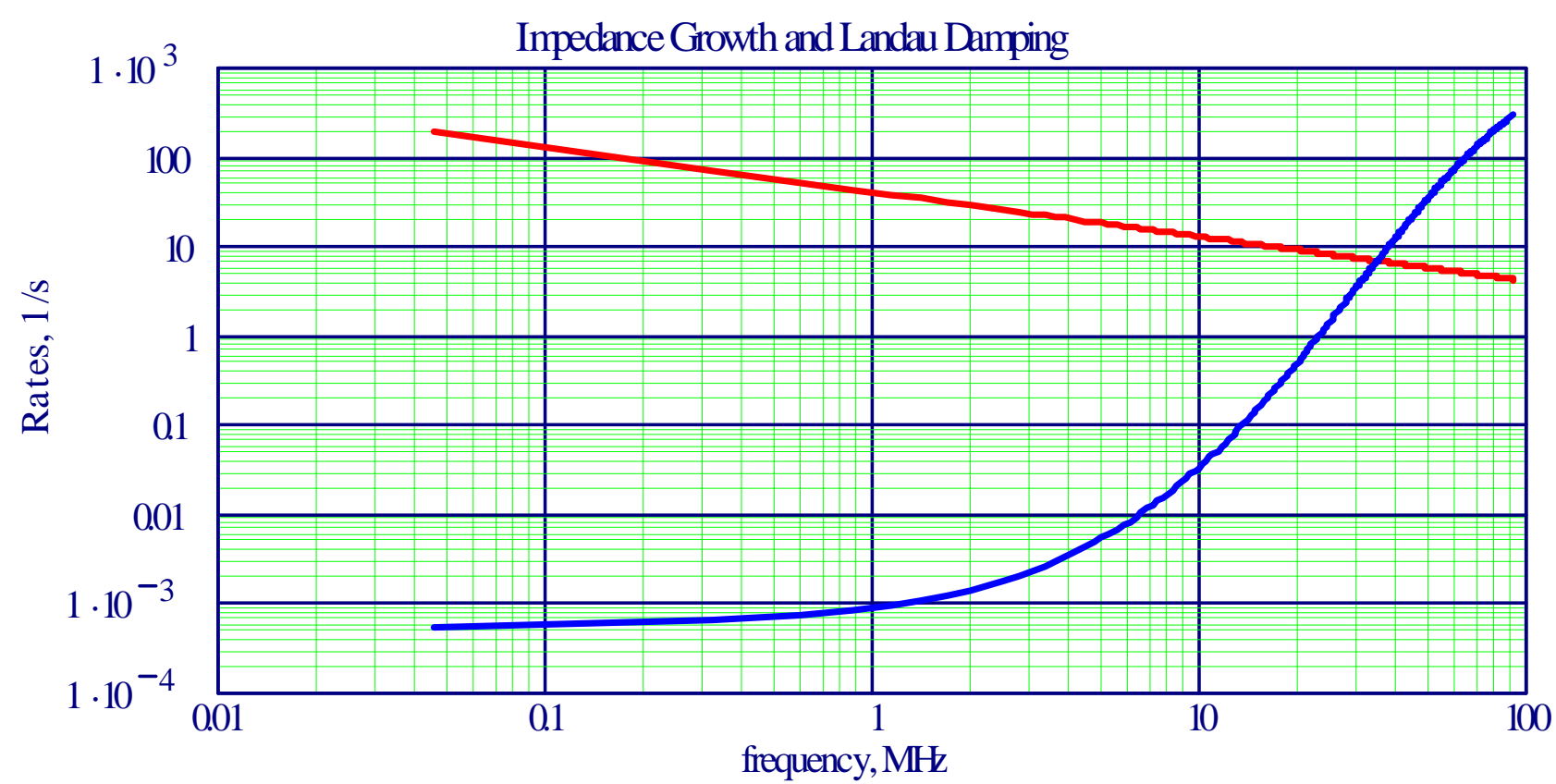

Figure 5: Instability growth (red) and Landau damping (blue) rates versus frequency. Without damper, perturbations above $35 \mathrm{MHz}$ are stable.

stability condition (4), this leads to increase of the threshold phase space density as $|n \eta-\xi|$, or $\simeq 20 \%$. Calculations in a manner of the previous subsections give for the threshold effective density $D=1.3$ in this case, perfectly confirming this conclusion.

\subsection{Pickup and Kicker}

Taking pickup and kicker voltages as

$$
V_{p}=\frac{N e l_{p}}{B c^{2} C_{p} b_{p}} y_{p}, \quad V_{k}=K(\omega) V_{p},
$$

where $C_{p}, l_{p}$ and $b_{p}$ are the pickup capacity, length and half-gap, $K(\omega)$ is the amplification, and $y_{p}$ is the beam offset at the pickup, the complex frequency shift introduced by the damper to the mode with average gradient $\bar{n}$ follows as

$$
\Delta \omega_{K}=-i \frac{N r_{0}}{2 \gamma B T_{0}} \frac{l_{p}}{C_{p} b_{p}} \frac{l_{k}}{b_{k}} \sqrt{\beta_{p} \beta_{k}} K\left(\omega_{b}+\bar{n} \omega\right)
$$

with $l_{k}$ and $b_{k}$ as the kicker length and half-gap, $\beta_{p, k}$ as the beta-functions at pickup (kicker) locations, and assuming $\pi / 2+\pi m$ for the pickup-kicker phase advance. The beam stability requires

$$
\operatorname{Im}\left(\Delta \omega_{K}+\Delta \omega_{Z}\right)<0
$$

for all the modes up to the threshold $\bar{n}=n_{t h}$, see Eqs. $(5,6)$.

Note that the definition of the amplification $K(\omega)(15)$ combines both the amplifier's gain $K_{a}(\omega)$ and the kicker response $K_{k}(\omega)$, or

$$
K(\omega)=K_{a}(\omega) K_{k}(\omega) .
$$

Assuming that the kicker is a strip-line of length $l_{k}=c \tau_{k}$, properly delayed, loaded (matched) with 50 $\Omega$ at the up-stream end and acting by the oncoming travelling wave, its response function is written: 

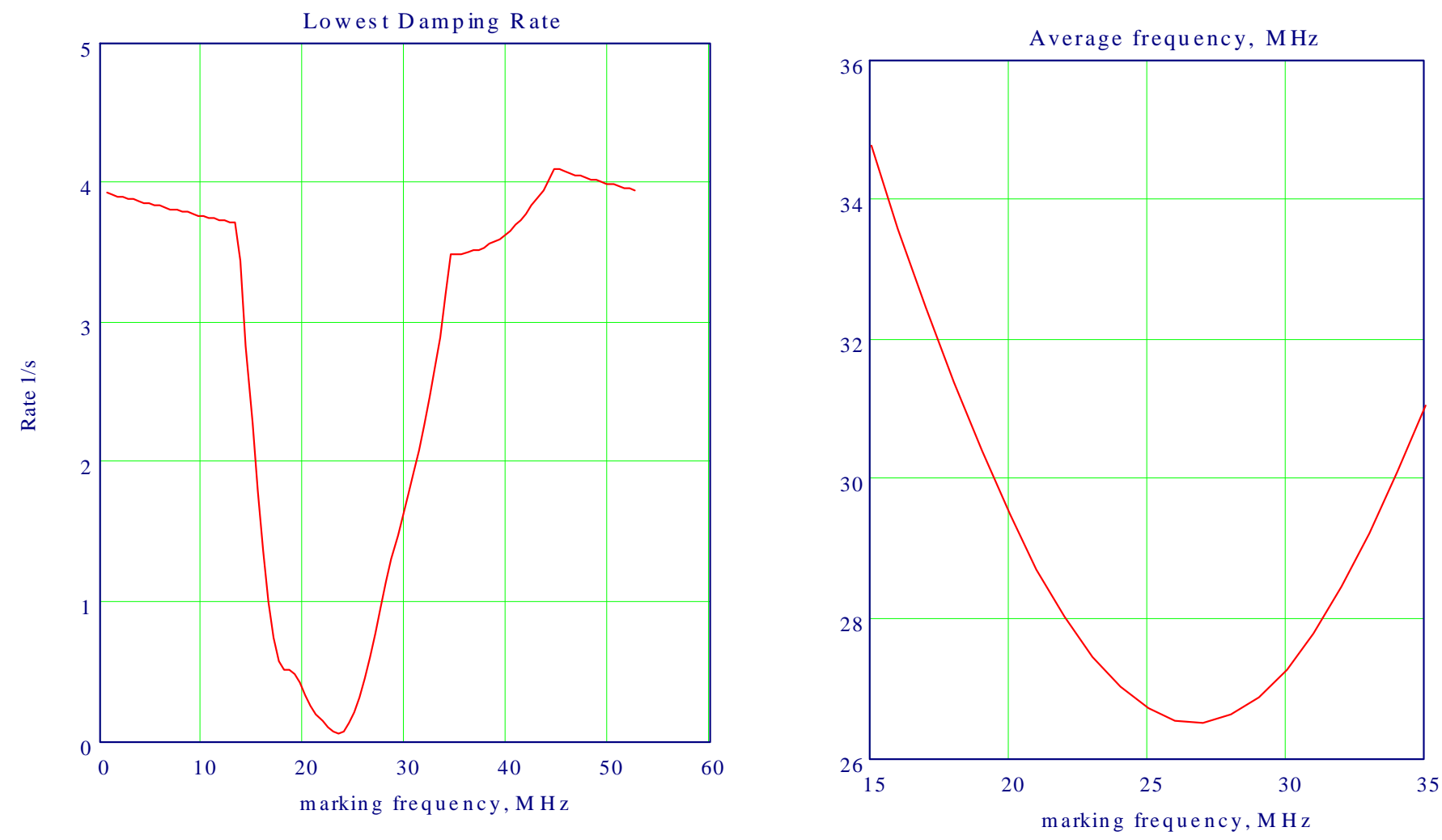

Figure 6: On the left: lowest damping rate for the system with ADT+LPF+Impedance+Landau damping. For the marking frequency $24 \mathrm{MHz}$ the rate is almost zero; thus, it is the threshold. On the right: average frequency of the perturbation as a function of the marking frequency for the less stable mode. 


$$
K_{k}(\omega)=\frac{\sin \left(\omega \tau_{k}\right)}{\omega \tau_{k}} .
$$

Current kicker is $l_{k}=1.4 \mathrm{~m}$ long; thus its response $K_{k}$ changes sign at $f_{k}=1 /\left(2 \tau_{k}\right)=107 \mathrm{MHz}$.

\section{Noise of the Damper}

Noise at the amplifier entrance at incoherent betatron frequencies leads to the beam emittance growth (normalized r. m. s.):

$$
\frac{d \varepsilon_{\perp}}{d t}=\int d \omega_{i} f\left(\omega_{i}\right) \sum_{n=-\infty}^{\infty} \frac{d J_{i, n}}{d t} \frac{1}{\left|\epsilon_{n, i}\right|^{2}} .
$$

Here the integral is taken over the individual betatron frequencies $\omega_{i}$, with $f\left(\omega_{i}\right)$ as the distribution function normalized by $\int d \omega_{i} f\left(\omega_{i}\right)=1$, and $d J_{i, n} / d t$ is a (bare) growth of an action of particle number $i$ due to the noise at frequency $\cong \omega_{b}+n \omega_{0}$ were this particle being alone (no beam response), while $\epsilon_{n, i}$ is the dielectric function, which takes into account the collective response (feedback through the beam) at frequency $\omega_{i}+n \omega_{0}$. Taking $U_{\omega}^{2}$ as the spectrum power for the noise voltage at the entrance of the amplifier, normalized as $\overline{U^{2}}=\int_{0}^{\infty} U_{\omega}^{2} d \omega / \pi$, the bare action growth follows:

$$
\frac{d J_{i, n}}{d t}=\frac{\gamma \beta_{k}}{2 T_{0}^{2}} \frac{l_{k}^{2}}{b_{k}^{2}} \frac{e^{2} U_{\omega}^{2}|K(\omega)|^{2}}{E_{0}^{2}} .
$$

The dielectric function can be presented as

$$
\epsilon_{n, i}=1+\left(\Delta \omega_{s c}+\Delta \omega_{Z}+\Delta \omega_{K}\right) \int \frac{f\left(\omega_{j}\right) d \omega_{j}}{\omega_{j}-\omega_{i}-i 0} .
$$

When the collective frequency stays outside the incoherent distribution, the contribution in the integral consists of two well-separated parts, from average and resonant particles:

$$
\begin{gathered}
\frac{d \varepsilon_{\perp}}{d t}=\left.\frac{d \varepsilon_{\perp}}{d t}\right|_{\mathrm{ave}}+\left.\frac{d \varepsilon_{\perp}}{d t}\right|_{\mathrm{res}} ; \\
\left.\frac{d \varepsilon_{\perp}}{d t}\right|_{\mathrm{ave}}=\left\langle\frac{1}{|\epsilon|^{2}}\right\rangle \sum_{n=-\infty}^{\infty} \frac{d J_{i, n}}{d t} \cong\left(\frac{\delta \omega}{\Delta \omega_{s c}}\right)^{2} \int d \omega_{i} f\left(\omega_{i}\right) \sum_{n=-\infty}^{\infty} \frac{d J_{i, n}}{d t} ; \\
\left.\frac{d \varepsilon_{\perp}}{d t}\right|_{\mathrm{res}}=\sum_{n=-\infty}^{\infty} \frac{d J_{i, n}}{d t} \frac{\Lambda_{L}}{\Lambda_{n}} .
\end{gathered}
$$

Here $\delta \omega$ is the betatron frequency width of the beam distribution, $\Lambda_{L} \cong \pi \Delta \omega_{s c}^{2} f\left(\Delta \omega_{s c}\right)$ is the Landau damping rate of the mode $n$, and $\Lambda_{n}=\Lambda_{L}-\operatorname{Im}\left(\Delta \omega_{Z}+\Delta \omega_{K}\right)$ is its total damping rate. For space charge dominated impedances, the beam frequency spread $\delta \omega$ is determined by the space charge itself due to the space charge transverse non-linearity. For Gaussian beams, this part of the spread is 0.25 of the average tune shift, which leads to suppression factor of the average contribution

$$
\left(\frac{\delta \omega}{\Delta \omega_{s c}}\right)^{2} \cong 0.06
$$

If the beam is not too close to the instability, the contribution of the resonant particles can be neglected compared with the average term, which leads to

$$
\frac{d \varepsilon_{\perp}}{d t} \simeq 0.06 \sum_{n=-\infty}^{\infty} \frac{d J_{i, n}}{d t}=0.06 \frac{\gamma \beta_{k}}{2 T_{0}} \frac{l_{k}^{2}}{b_{k}^{2}} \frac{e^{2}}{E_{0}^{2}} \int_{0}^{\infty} \frac{d \omega}{\pi} U_{\omega}^{2}|K(\omega)|^{2}
$$




\section{High Frequency Damper}

\subsection{Analog Damper}

To accumulate $6 \cdot 10^{12}$ antiprotons in $30 \mathrm{eVs}$ and $5 \mathrm{~mm} \mathrm{mrad}$ of $95 \%$ emittances corresponds to the effective density $D=4$, which is about 4 times higher than the threshold with the existing damper. Thus, this goal requires significant extension of the damper bandwidth. A possible solution could be an analog damper based on on a first-order filter (14). Fig. (4.1) shows the impedance-driven growth, Landau damping and Landau damping plus the high frequency analog damper for the mentioned goal beam parameters and the first-order damper with the roll-off frequency $\omega_{F} /(2 \pi)=26 \mathrm{MHz}$ and low-frequency damping time of 150 revolutions. According to the plot, the beam is close to the threshold. Several important features have to be underlined here.

- Landau damping prevails starting from $250 \mathrm{MHz}$. Below this frequency, damper is the only remedy.

- Thus, the damper is and must be effective up to an order of magnitude of the roll-off frequency.

- The pickup and the kicker have to be good up to $250 \mathrm{MHz}$.

- Filter of the first order is the best one (assuming that filters of the order less than 1 do not exist).

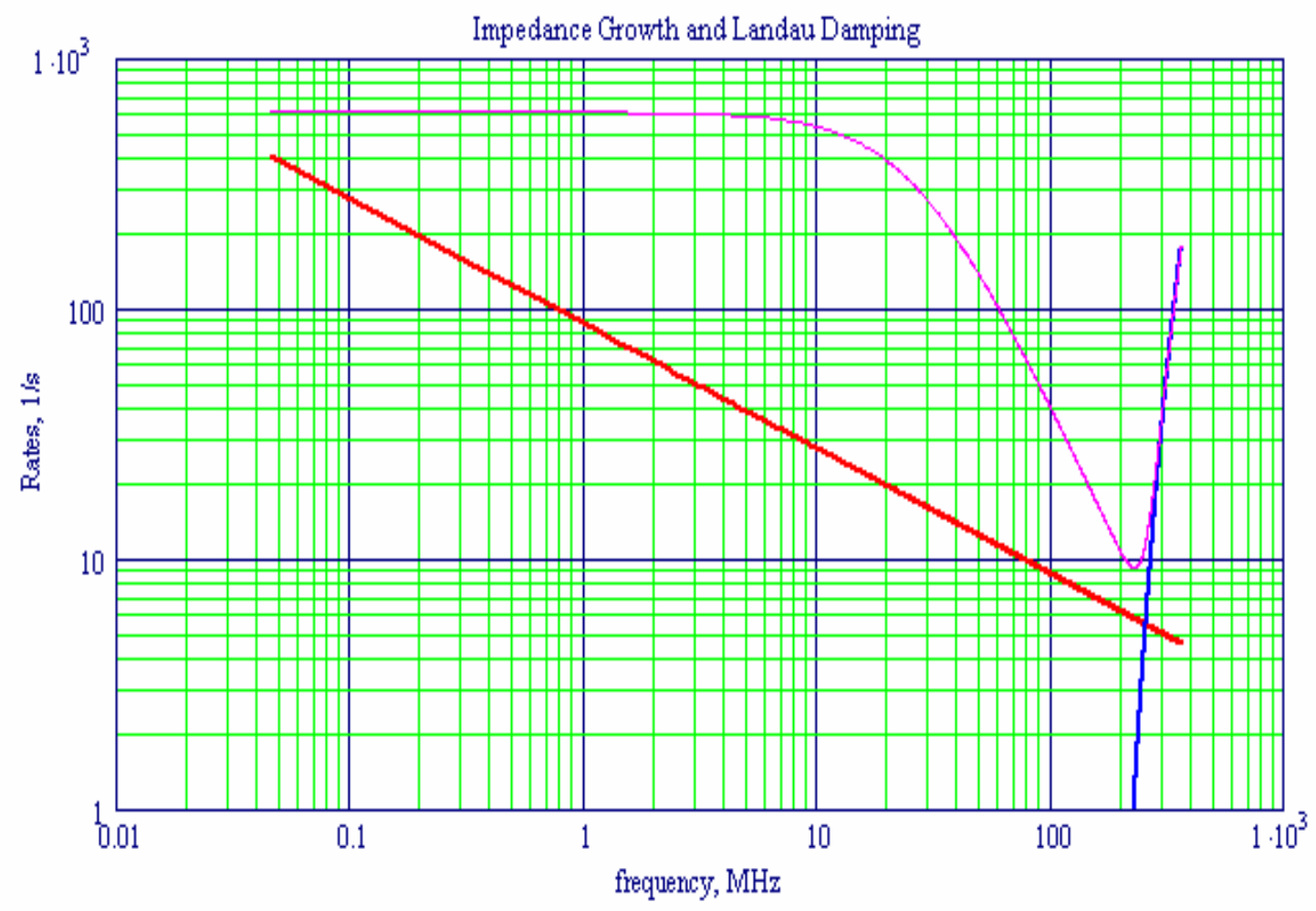

Impedance-related growth (red), Landau damping (blue) and Landau damping plus high frequency analog damper (magenta) rates

More optimal way for damping optimization requires use of both existing low-frequency digital damper and high-frequency analog one. In this case, the resulting damping rate could better fit the impedance growth rate. A possible solution with the analog damper roll-off at $100 \mathrm{MHz}$ and low-frequency damping time of 3000 turns is shown in Figs. (7), (8), showing that at these parameters the beam is at the instability threshold. 


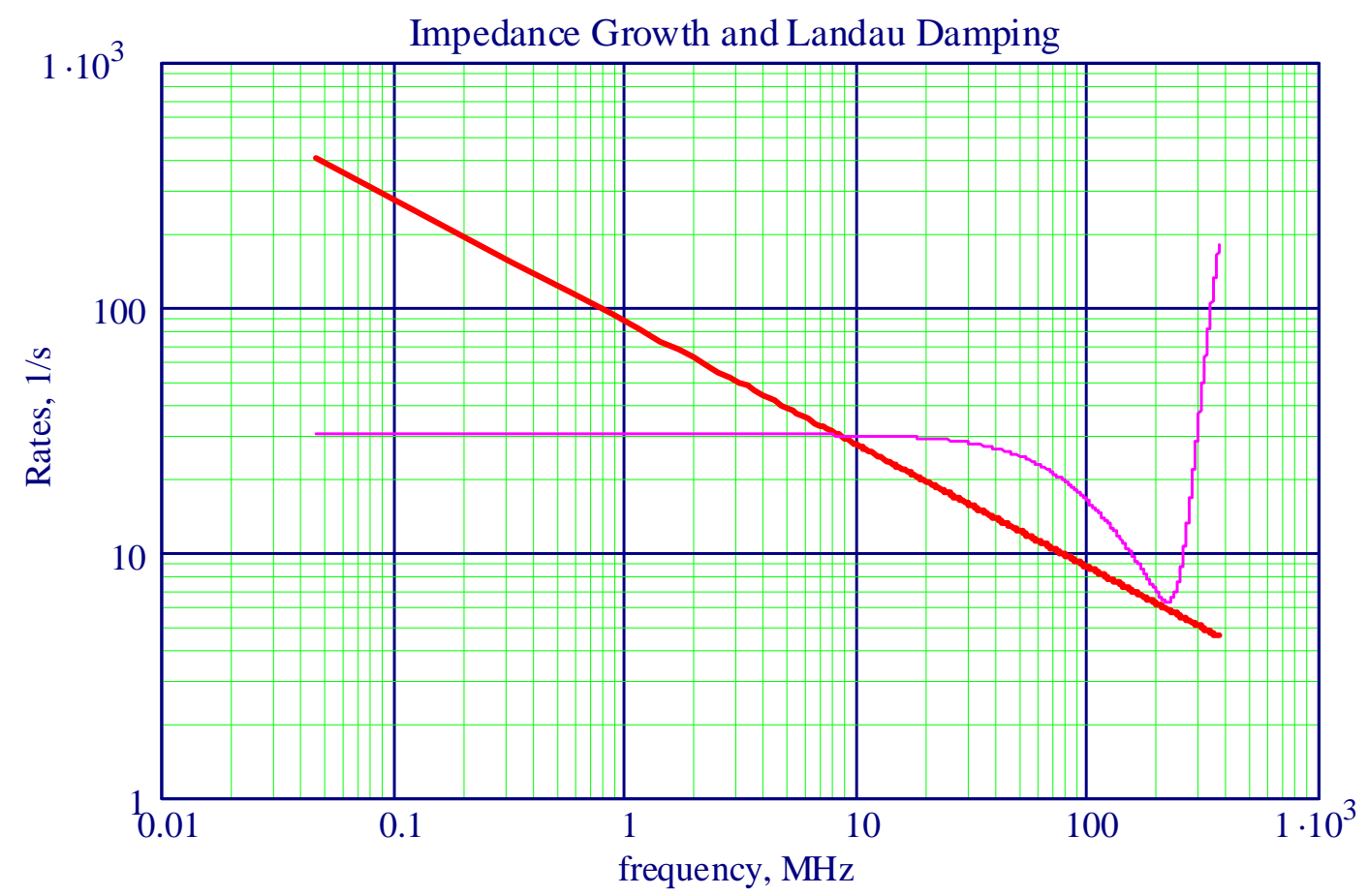

Figure 7: Impedance-related growth (red) and Landau damping plus 2nd stage high frequency analog damper (magenta) rates

\subsection{Increase of the Sampling Rate}

When this paper was prepared, the sampling rate of the digital damper was increased twice, from 53 to 106 MHz. Application of the described analysis requires the kicker frequency response being taken into account. This doubling of the sample rate further increases the threshold, calculated for the same other conditions as $D=1.4$ with the LPF, and slightly more, $D=1.5$, without the LPF. Both results are not sensitive to the averaging parameter $N_{a}$.

Within a few days from a day when this is written, one more doubling of the sample rate will be effective. With the current kicker, however, increase of the sample rate above $106 \mathrm{MHz}$ is seen as useless at best. With an ideal kicker and the sampling rate of $212 \mathrm{MHz}$, the threshold goes up to $D=2.5$, and the analog damper will not, probably, be needed with this scheme.

\section{Summary}

Instability in the Recycler is driven by the resistive wall impedance, while the Landau decrement rate is suppressed by the space charge. The quantitative stability analysis is presented where the mentioned factors and the damper are taken into account. The stability thresholds are calculated for various damper options. Requirements for the high-frequency damper are specified. Quadrupling of the sample frequency and 3-4 times increase of the kicker's band makes the analog high frequency damper hardly necessary.

The momentum distribution was assumed to be Gaussian in this paper, which is not quite true for ecooled beams, which have normally somewhat higher tails. From this point of view, the stability requirements found here could probably be slightly relaxed.

The author is thankful to V. Lebedev and S. Nagaitsev for many enlightening ideas, to M. Hu for his description of the instability observations, and to J. Crisp for the data and the explanation about technical features of the digital damper. 

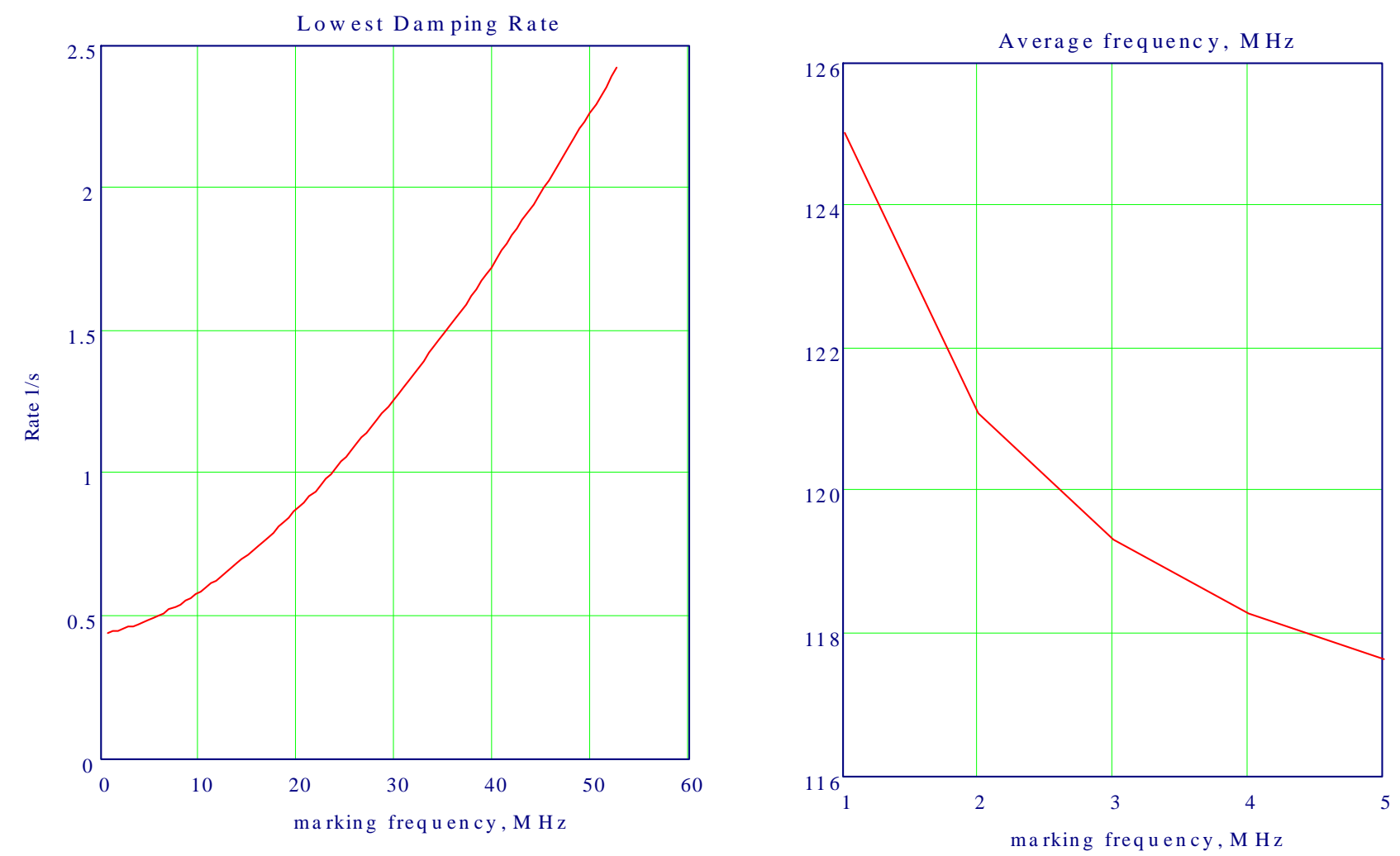

Figure 8: Total damping rate of the least stable mode versus the marking frequency (left), and the average frequency for this mode (right). 


\section{References}

[1] A. W. Chao, "Physics of Collective Beam Instabilities in High Energy Accelerators", J. Wiley \& Sons, Inc., 1993.

[2] V. Lebedev, private communication, March 2005

[3] V. Balbekov, Proc. PAC'05

[4] M. Hu, private communication, Oct. 2005.

[5] J. Crisp, private communication, Oct. 2005. 